\title{
Epoxidation and Deoxygenation of Single-Walled Carbon Nanotubes: Quantification of Epoxide Defects
}

\author{
Douglas Ogrin, Jayanta Chattopadhyay, Anil K. Sadana, W. Edward Billups, * \\ and Andrew R. Barron* \\ Department of Chemistry and Center for Nanoscale Science and Technology, \\ Rice University, Houston, Texas 77005
}

Supplementary Materials 


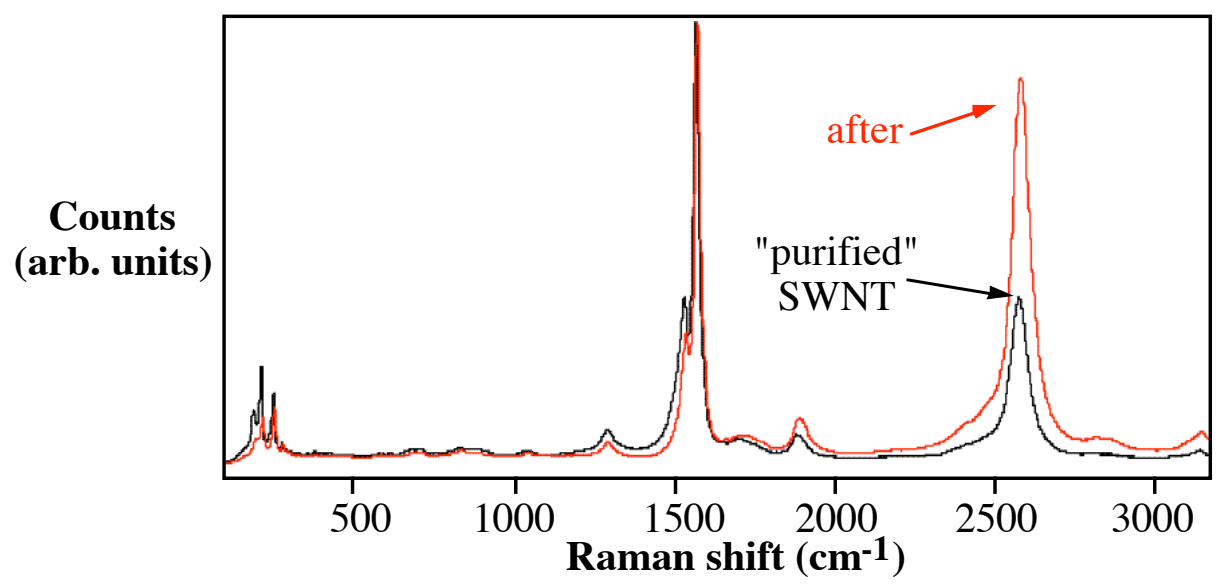

Figure S1. Raman spectra (633 nm excitation) of $\mathrm{SF}_{6}$ purified SWNTs before (black) and after (red) deoxygenation with excess $\mathrm{MTO} / \mathrm{PPh}_{3}$. 
(a)

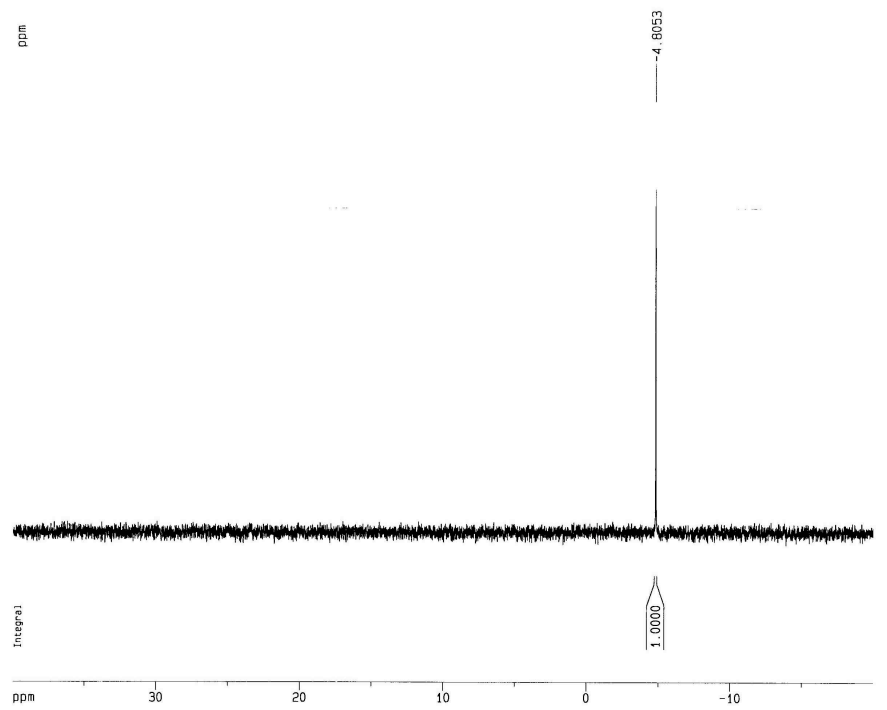

(b)

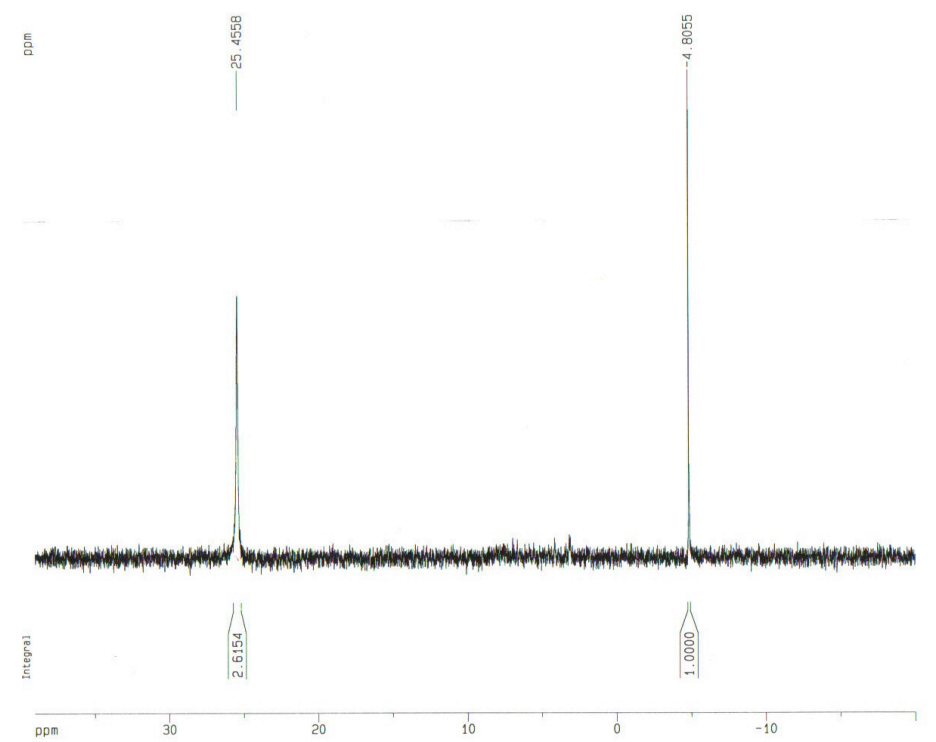

Figure S2. ${ }^{31} \mathrm{P}\left\{{ }^{1} \mathrm{H}\right\}$ NMR spectra of (a) the initial $\mathrm{MTO} / \mathrm{PPh}_{3} / \mathrm{SWNT}$ solution and (b) after $17 \mathrm{~h}$. 
(a)
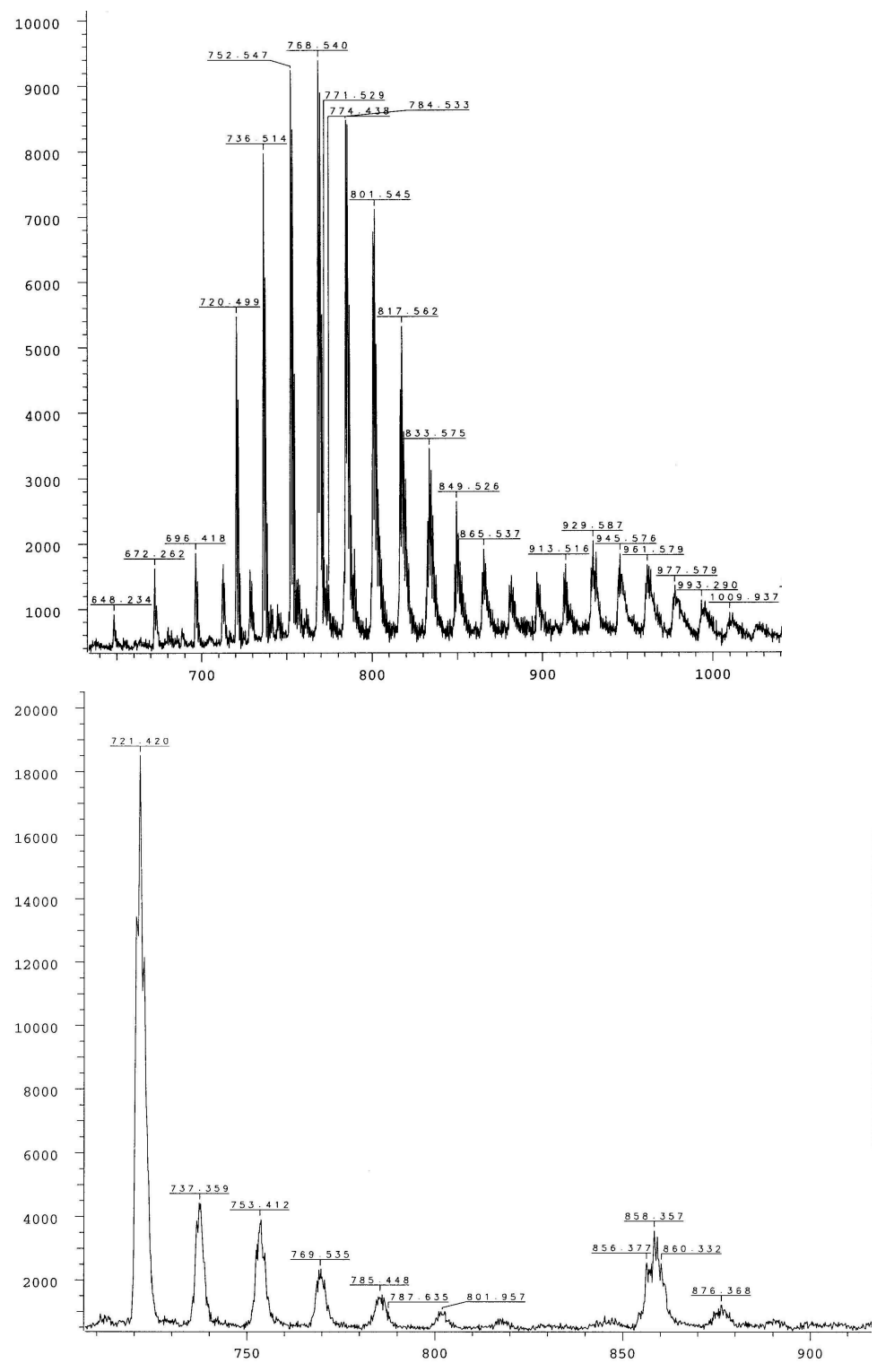

(b)

Figure S3. Mass Spectrum of product distribution of $\mathrm{C}_{60}(\mathrm{O})_{\mathrm{x}}$ before (a) and after (b) reaction with $\mathrm{MTO} / \mathrm{PPh}_{3}$. 


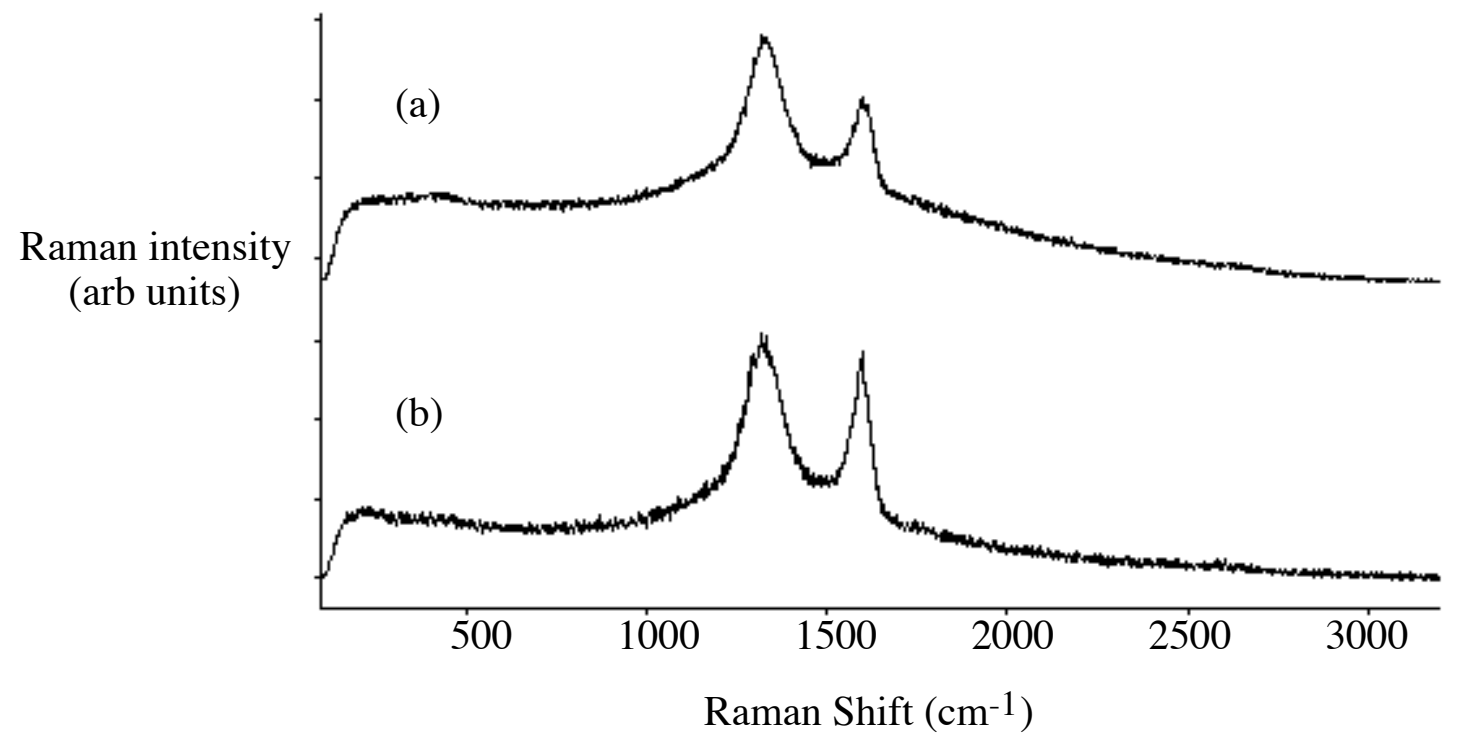

Figure S4. Raman spectra (780 nm) of epoxidized SWNTs using $m$-CPBA at (a) room temperature and (b) $50{ }^{\circ} \mathrm{C}$. 


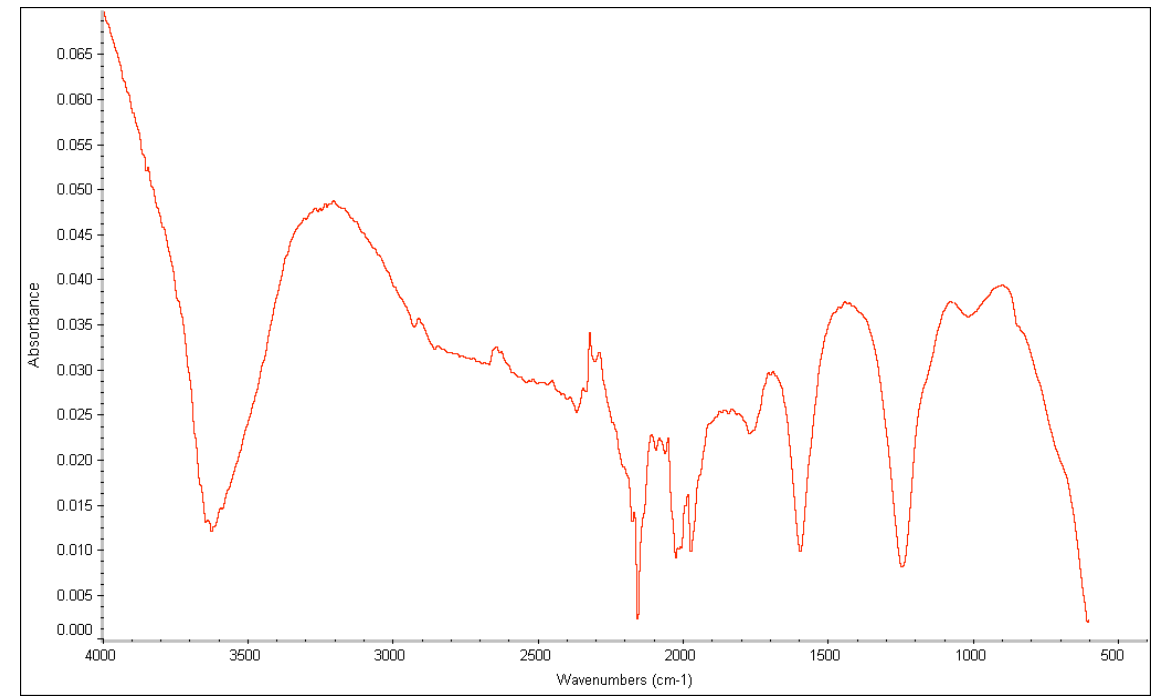

Figure S5. FT-IR of epoxidized SWNTs using trifluorodimethyldioxirane. 


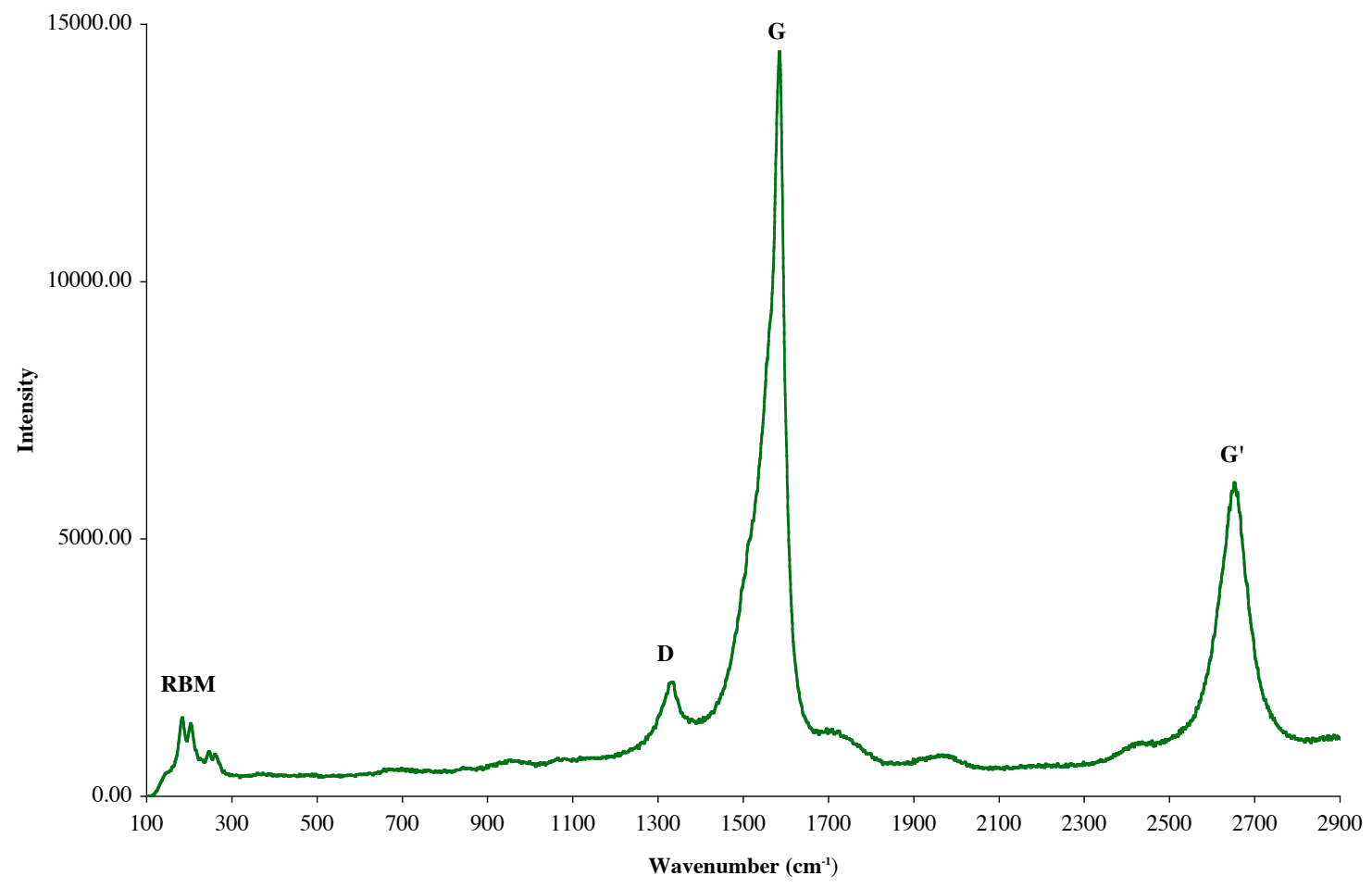

Figure S6. Raman spectra of O-SWNTs after TGA using $532 \mathrm{~nm}$ excitation. 


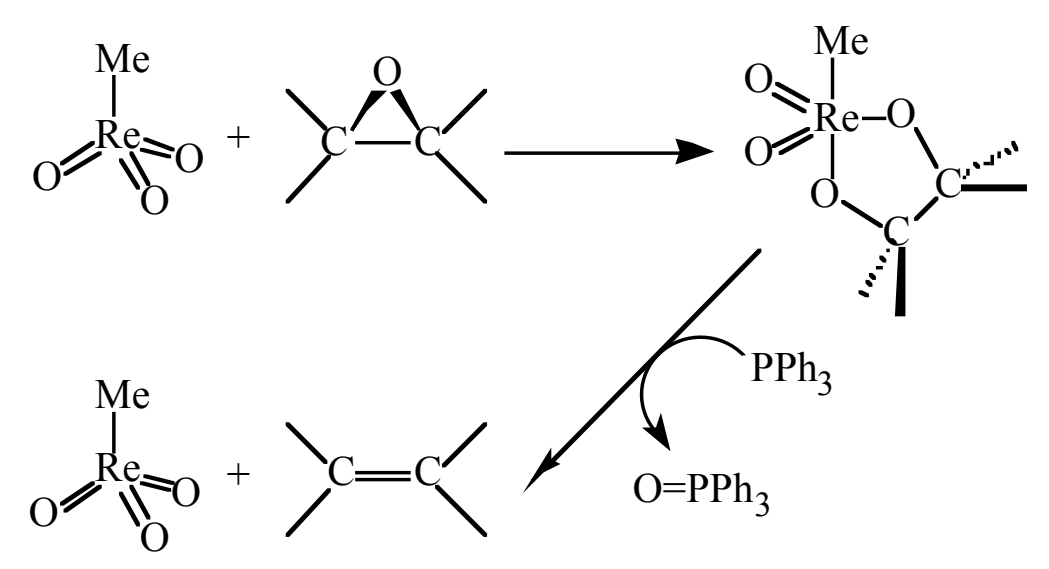

Scheme S1. MTO deoxygenation of an olefin. 